\title{
Investigation of a family cluster outbreak of COVID-19 indicates the necessity of CT screening for asymptomatic family members in close contact with confirmed patients
}

\author{
Zhifeng Liu ${ }^{1 \#}$, Qiwen Wu ${ }^{1 \#}$, Zhikang Zou ${ }^{1}$, Yeping Yao ${ }^{1}$, Jinhui Cai ${ }^{1}$, Tao Liu ${ }^{2}$, Zhengfei Yang ${ }^{1,3}$, \\ Qingyu Liu ${ }^{1,3}$ \\ ${ }^{1}$ Zengcheng District People's Hospital of Guangzhou, Guangzhou, China; ${ }^{2}$ Brown University, Providence, RI, USA; ${ }^{3}$ Sun Yat-sen Memorial \\ Hospital, Sun Yat-sen University, Guangzhou, China \\ Contributions: (I) Conception and design: Z Yang, Q Liu; (II) Administrative support: J Cai, T Liu; (III) Provision of study materials or patients: Z \\ Liu, Q Wu; (IV) Collection and assembly of data: Z Liu, Q Wu, Z Zou, Y Yao; (V) Data analysis and interpretation: Z Liu, Q Wu, Z Yang, Q Liu; (VI) \\ Manuscript writing: All authors; (VII) Final approval of manuscript: All authors. \\ \#These authors contributed equally to this work. \\ Correspondence to: Qingyu Liu, MD, PhD; Zhengfei Yang, MD, PhD. Sun Yat-sen Memorial Hospital, Sun Yat-sen University, 107 Yan Jiang Xi Road, \\ Guangzhou 510120, China. Email: liu.qingyu@163.com; yangzhengfei@vip.163.com.
}

Background: To investigate whether asymptomatic close-contact family members of patients diagnosed with coronavirus disease (COVID-19) should immediately undergo CT screening in addition to the viral nucleic acid test.

Methods: We retrospectively analyzed the data of a family cluster of 8 individuals, of whom 1 family member (Patient 3) had an epidemiologic history of having visited Guangzhou from Hubei Province on January 20, 2020. Her father (Patient 1) developed a fever and respiratory system symptoms and was confirmed COVID-19-positive on February 4-5, 2020 at Zengcheng People's Hospital, Guangzhou, China. Seven close-contact family members of the patients were then screened for COVID-19 on February 5-6 at the hospital. The CT imaging manifestation and laboratory tests of this family cluster were investigated and reported.

Results: Five (62.5\%) of the 8 family members were confirmed COVID-19-positive. Except for Patient 1, who had fever, cough, fatigue, and dizziness, the remaining four (4/5, 80\%) COVID-19-positive family members (Patients 2-5) had no clinical symptoms. Among the 5 patients, 2 had leukopenia (2/5, 40\%), 1 had low absolute neutrophil counts $(1 / 5,20 \%)$, and 2 had increased high-sensitivity C-reactive protein $(2 / 5$, $40 \%)$. Ground-glass opacity (GGO) was found on chest CT imaging in all 5 patients $(5 / 5,100 \%)$, with interlobular septal thickening. Thickened blood vessel shadows were seen in 3 patients (3/5, 60\%). The 3 COVID-19-negative family members (Family Members 1-3) did not have CT abnormalities, and they showed negative reverse transcription-polymerase chain reaction (RT-PCR) results twice.

Conclusions: CT screening is necessary in close-contact family members of a confirmed COVID-19 pneumonia case, regardless of the presence of clinical symptoms.

Keywords: 2019-novel coronavirus (2019-nCoV); coronavirus disease (COVID-19); pneumonia; computed tomography

Submitted Feb 19, 2020. Accepted for publication May 30, 2020.

doi: $10.21037 /$ jtd-20-955

View this article at: http://dx.doi.org/10.21037/jtd-20-955 


\section{Introduction}

Since December 2019, multiple cases of unexplained pneumonia have been found, there was a novel coronavirus was detected by the laboratory on January 7, 2020, and the entire genome sequence of the virus was obtained $(1,2)$. The World Health Organization (WHO) tentatively named the new coronavirus "2019-novel coronavirus" (2019-nCoV) on January 12, and formally named the coronavirus disease "COVID-19" on February $11(3,4)$. As of February 18, 2020, 72,436 cases have confirmed COVID-19, and including 1,868 deaths (5).

Symptoms of COVID-19 include fever, dry cough, shortness of breath, and dyspnea (3). Severe cases can cause severe acute respiratory syndrome, kidney failure, and even death $(6,7)$. Previous research has found that imported and clustered outbreaks have occurred outside Hubei Province, especially familial cluster outbreak $(8,9)$. Chan et al. (8) reported that 5 members of a family in Shenzhen who traveled to Wuhan were diagnosed with COVID-19. They developed symptoms such as fever, cough, and diarrhea, and another family member who did not go to Wuhan was also confirmed to have been infected by human-to-human transmission. Early detection of 2019-nCoV infection in individuals who was in close contact with patients diagnosed with COVID-19 pneumonia is important to prevent further spread of the epidemic (9-11).

In the present study, we retrospectively review a familial cluster of COVID-19 pneumonia in a Guangzhou family with a history of contact in Hubei. Five of the 8 family members were confirmed to have COVID-19 pneumonia by reverse transcription-polymerase chain reaction (RT-PCR). Four of these patients did not have any clinical symptoms related to COVID-19 pneumonia, but their lung CT manifestations showed ground-glass opacity (GGO) lesions. The epidemiologic histories, symptoms, laboratory tests and CT imaging data in this family cluster were retrospectively analyzed, and the purpose of our study is to suggest the necessity of CT screening of family members who was in close contact with confirmed COVID-19 pneumonia patients.

\section{Methods}

\section{Patient information}

This study was conducted in accordance with a protocol approved by the Research Ethics Committee of Zengcheng District People's Hospital of Guangzhou. We retrospectively analyzed clinical data of 8 close-contact family members at Zengcheng People's Hospital in Guangzhou from February 4 to 6, 2020, of whom 5 were confirmed to have COVID-19 pneumonia (Patients 1-5), and the remaining 3 tested negative (Family Members 1-3). Their epidemiological, clinical and laboratory data were collected.

\section{Family information}

Patients 1 and 2 are a couple, Patient 3 is their elder daughter, Patient 4 is the younger daughter, and Patient 5 is Patient 4's husband. Family Member 1 is Patient 3's husband and Family Member 2 is their son. Family Member 3 is the son of Patients 4 and 5. Patient 3 traveled with Family Members 1 and 2 from Hubei Province to Guangzhou to meet with the 5 other family members on January 20, 2020. On January 31, 2020, Patient 1 developed a cough. He developed weakness after 2 days, dizziness occurred after 3 days, fever $\left(37.5^{\circ} \mathrm{C}\right)$ occurred after 4 days, and he ultimately went to the hospital for treatment. On February 5, his RT-PCR nucleic acid test result was positive for 2019-nCoV, and he was the first confirmed COVID-19 pneumonia family member. At the same time, the patient's 7 close family members described above were screened for COVID-19. Their CT examinations revealed that 4 of them had GGO lesions in their lungs and RT-PCR nucleic acid test results were positive (Figure 1).

\section{CT scan protocol}

Eight family members were used a 128 -slice spiral CT unit (SOMATOM, Definition AS, Siemens Healthineers, Germany) for chest scans ranging from the apex to the bottom of the lung. The supine position was scanned from the patient's head to the foot. Scanning parameters were as follows: tube voltage $120 \mathrm{kV}$, automatic tube current $110 \mathrm{~mA}$, collimator width $64 \mathrm{~mm}$, rack rotation time $0.5 \mathrm{~s} / \mathrm{r}$, pitch 1.2 , scanning field of view $255 \mathrm{~mm} \times 255 \mathrm{~mm}$, scanning time $3.98 \mathrm{~s}$, and thickness $1.0 \mathrm{~mm}$ images for reconstruction.

\section{CT image analysis}

CT findings were analyzed by two radiologists with more than 10 years of experience on the $1 \mathrm{~mm}$ thickness CT images. The parameters for viewing $\mathrm{CT}$ images were as follows: for the lung window, a window width of 1,500 to $2,000 \mathrm{HU}$ and window level of -450 to $-600 \mathrm{HU}$; 

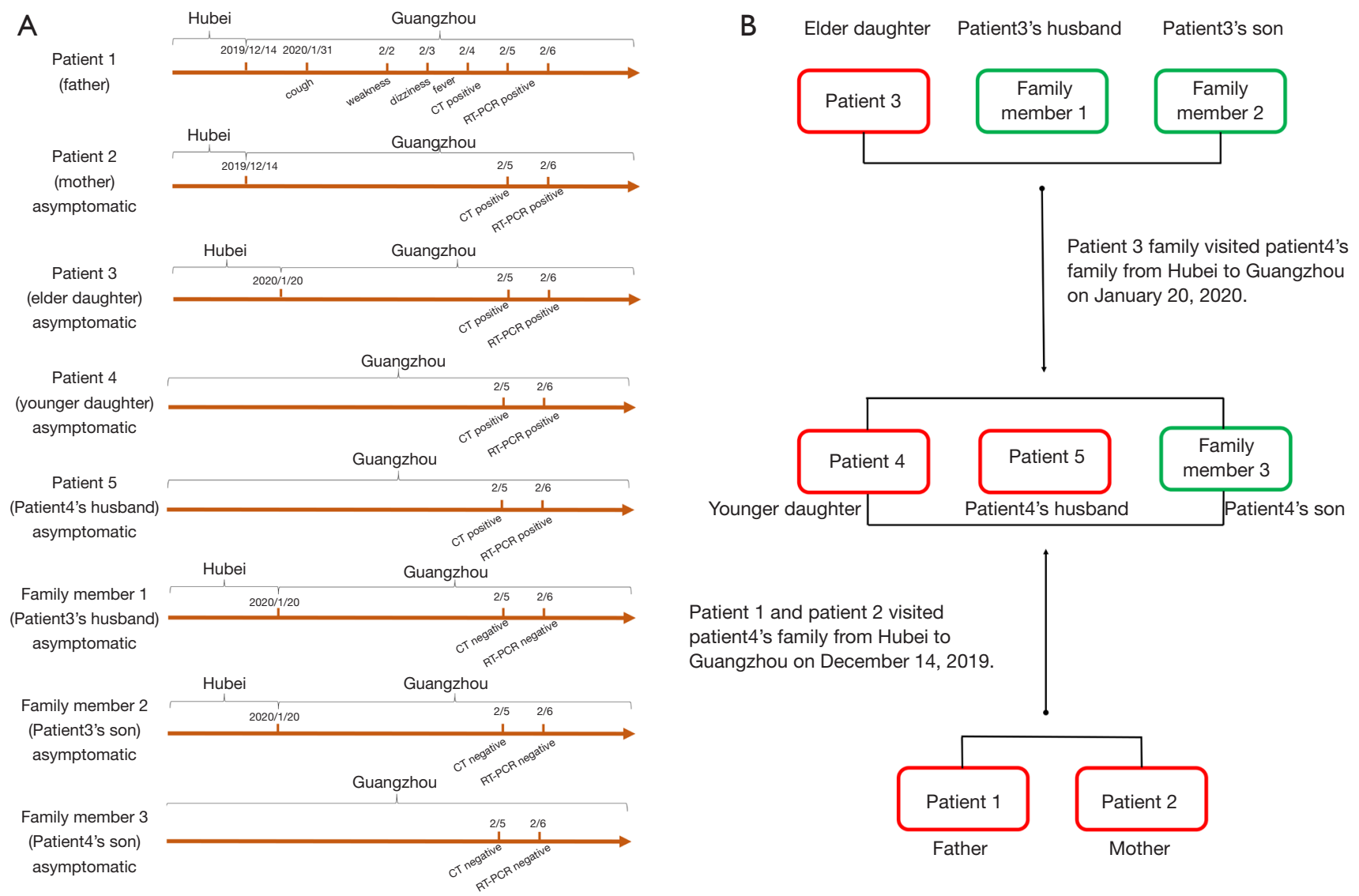

Figure 1 An epidemiological timeline and relationship diagram of a family clustering. (A) The epidemiologic trajectories of five confirmed COVID-19 pneumonia patients and the other three family members, the time of first clinical symptoms, the time of CT examination results, and the time of RT-PCR test results. (B) Pedigree plot of five confirmed COVID-19 pneumonia patients and the other three family members. RT-PCR, reverse transcription-polymerase chain reaction.

for the mediastinum window, a window width of 250 to $350 \mathrm{HU}$ and window level of 30 to $50 \mathrm{HU}$. They analyzed the patients' CT images for GGO, consolidation, interlobular septum, thickened blood vessels, pleural effusion, and pericardial effusion, and the distribution of lung abnormalities.

\section{RT-PCR test}

COVID-19 nucleic acid testing was performed using the Viral Nucleic Acid Kit (Health, Ningbo, China) to extract nucleic acids according to the CCDC recommended kit instructions. A COVID-19 detection kit (Bioperfectus, Taizhou, China) was used to detect the ORF1ab gene (nCovORF1ab) and the $N$ gene (nCoV-NP) according to the manufacturer's instructions using real-time RT-PCR. If both the $\mathrm{nCovORF} 1 \mathrm{ab}$ and $\mathrm{nCoV}-\mathrm{NP}$ showed positive results, the COVID-19 infection was considered.

\section{Statistical analysis}

Statistical analysis was done with SPSS (version 25.0). Continuous variables were expressed as mean and range. Categorical variables were expressed as count and percentage (\%).

\section{Results}

Five of the 8 family members were confirmed to have COVID-19 pneumonia $(5 / 8,62.5 \%)$. The 5 patients had an average age of 53.6 years (range, 41 to 72 years). There were 2 males and 3 females. The 3 COVID-19-negative family members had an average age of 27 years (range, 15 
to 47 years). Only Patient $1(1 / 5,20 \%)$ of the 5 confirmed COVID-19 pneumonia cases developed fever, cough, fatigue, and dizziness. The remaining 4 patients $(4 / 5,80 \%)$ did not show any clinical symptoms (Table 1). CT results of the 5 confirmed COVID-19 pneumonia patients were all positive $(5 / 5,100 \%)$, and the CT results of the other 3 close-contact family members with negative nucleic acid tests (twice) for 2019-nCoV were normal.

\section{Epidemiologic history}

Patients 1 and 2 returned to Guangzhou from Hubei on December 14, 2019, and Patient 3, Family Member 1, and Family Member 2 returned to Guangzhou from Hubei on January 20. Patient 4, Patient 5, and Family Member 3 did not travel to Hubei, whom live in Guangzhou all year round. Details of their epidemiologic history, the time of clinical symptoms, the time of CT examination results, and the time of RT-PCR results of the 5 confirmed COVID-19 pneumonia patients and the other 3 negative family members are depicted in Figure 1.

\section{Laboratory results}

Among the 5 patients confirmed to have COVID-19 pneumonia, 2 patients had leukopenia $(2 / 5,40 \%), 1$ patient had decreased neutrophil counts $(1 / 5,20 \%)$, and 2 patients had increased high-sensitivity C-reactive protein (2/5, $40 \%)$. Their neutrophil counts, percentage of lymphocytes, absolute number of lymphocytes, procalcitonin levels and 9 respiratory items (legionella pneumophila, mycoplasma pneumoniae, chlamydia pneumoniae, adenovirus, respiratory syncytial virus, influenza $A$ virus, influenza $B$ virus, parainfluenza virus, and $\mathrm{Q}$ fever rickettsia) are shown in Table 1.

\section{CT image analysis}

CT images of the 5 COVID-19 pneumonia patients showed lobular, segmental, or subsegmental lung abnormalities, and the subpleural areas were the most involved. Bilateral lung abnormalities were found in 4 cases $(4 / 5,80 \%)$ and unilateral lung abnormalities were found in 1 case $(1 / 5$, $20 \%)$. GGO lesions $(5 / 5,100 \%)$ and interlobular septal thickening $(5 / 5,100 \%)$ were found on the CT images of the 5 patients (Figures 2-6). In addition, CT images of 3 patients showed signs of thickened blood vessels in GGO lesion $(3 / 5,60 \%$ ) (Figure $2 A$ ). No pleural effusion or pericardial effusion was found on the CT images of any of the 5 patients (Table 1).

\section{Follow up}

These 5 patients with COVID-19 pneumonia were followed up for two weeks, and one patient's condition (Patient 1) deteriorated, one patient (Patient 5) developed cough on the second day after transfer to the designated hospital, CT results from those two patients showed increased GGO lesions. Other asymptomatic patients (Patients 2-4) were still asymptomatic, and lung abnormalities did not show progress on CT scans.

\section{Discussion}

We retrospectively analyzed a familial cluster of 5 cases of COVID-19 pneumonia. Emergency CT images of Patient 1 revealed multiple GGO lesions in both lungs. Laboratory results showed that the patient's absolute counts of white blood cells and lymphocytes were decreased, and levels of high-sensitivity C-reactive protein were increased. On February 5, the patient's RT-PCR nucleic acid test results were positive for $2019-\mathrm{nCoV}$, and he was the first confirmed COVID-19 pneumonia case in the family. On the same day, 7 close family members of the patients were screened for COVID-19 with laboratory test and CT scan. Their CT examinations revealed that 4 of them (Patients 2-5) had GGO lesions in the lungs and RT-PCR nucleic acid test results were positive for 2019-nCoV, although they did not show any typical clinical symptoms of fever, cough, fatigue, or sore throat, etc. Asymptomatic COVID-19 pneumonia patients may become an important source of infection and increase the difficulty in controlling the outbreak, highlighting the importance to screen early and quickly for this asymptomatic COVID-19 patients who was in close contact with confirmed case in a familial cluster.

In this study case, there were 2 Hubei historical time points. The first time point was December 14, 2019. Patients 1 and 2 traveled from Hubei to Guangzhou to meet with Patient 4, Patient 5, and Family Member 3. The second time point was January 20, 2020, when Patient 3 traveled with Family Members 1 and 2 from Hubei to Guangzhou to meet with the 5 other family members. Patient 1 began to have clinical symptoms 49 days from the first time point and 11 days from the second time point. According to recent studies, the longest reported incubation period for COVID-19 pneumonia patients was 24 days, and 


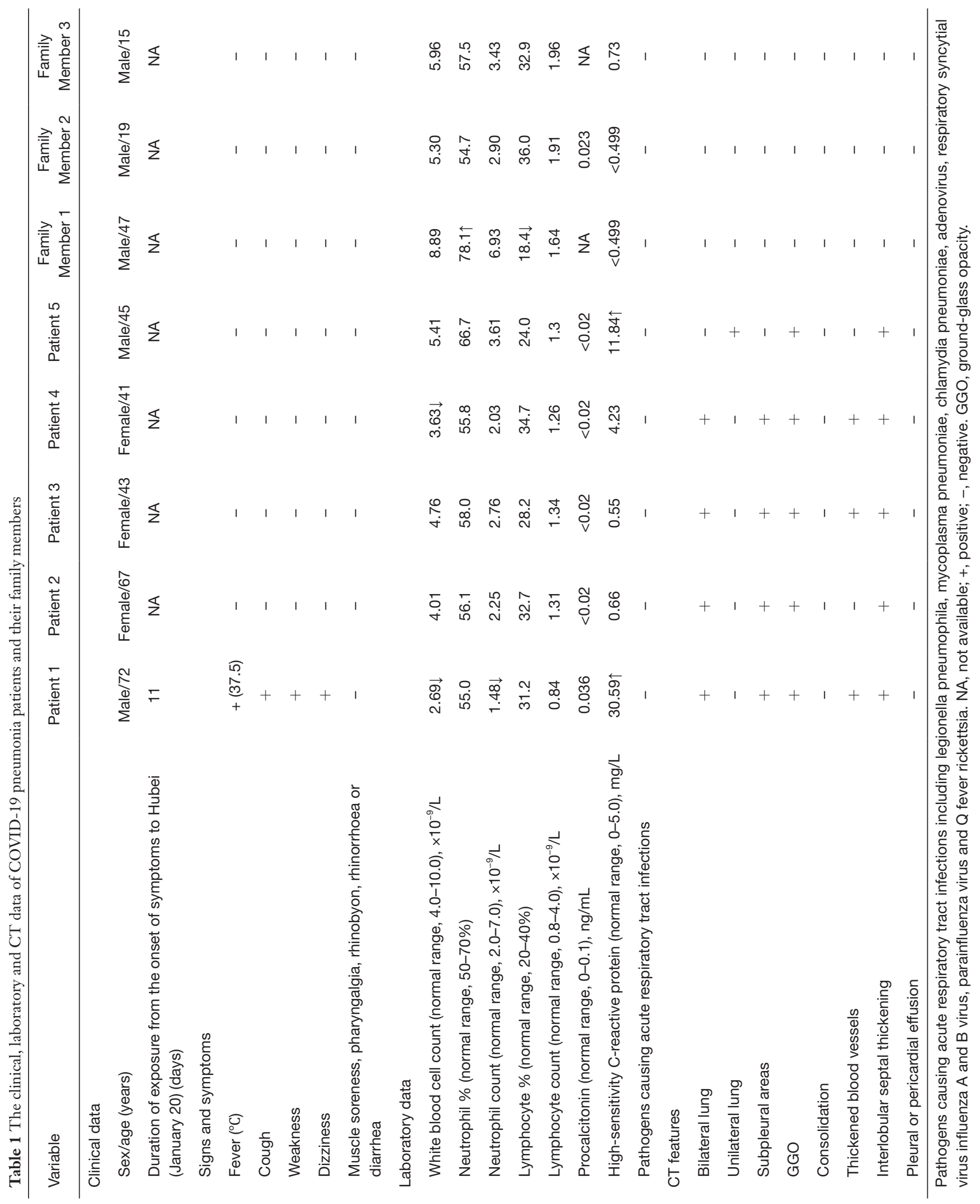



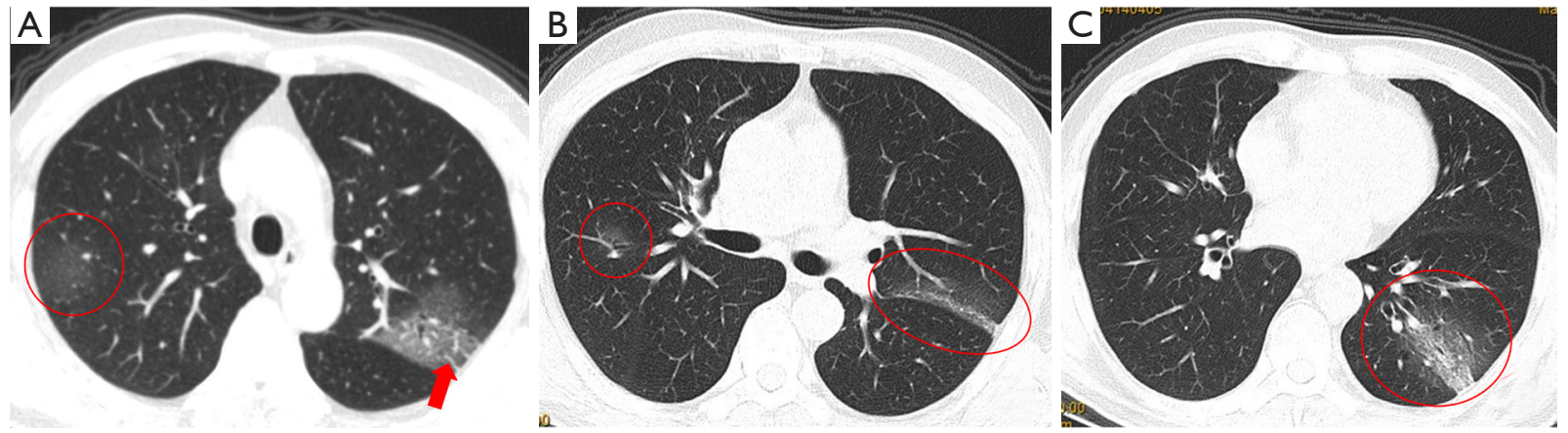

Figure 2 Patient 1, male, 72 years old. CT images showed multiple subpleural GGOs in bilateral lung segmental and subsegmental regions (A,B,C, red circles). Thickened blood vessels and interlobular septum thickening were seen in the lesion (A, red arrow). GGO, ground-glass opacity.
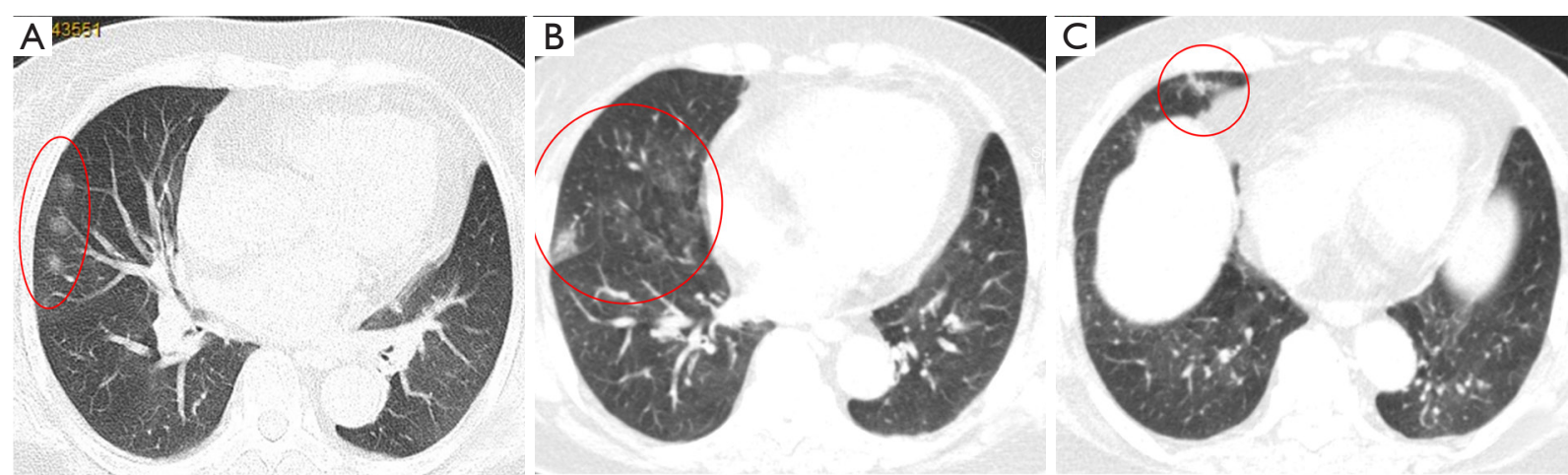

Figure 3 Patient 2, female, 67 years old. CT images showed multiple sub-segmental small-scale GGOs in the bilateral subpleural region (A,B,C, red circle). GGO, ground-glass opacity.
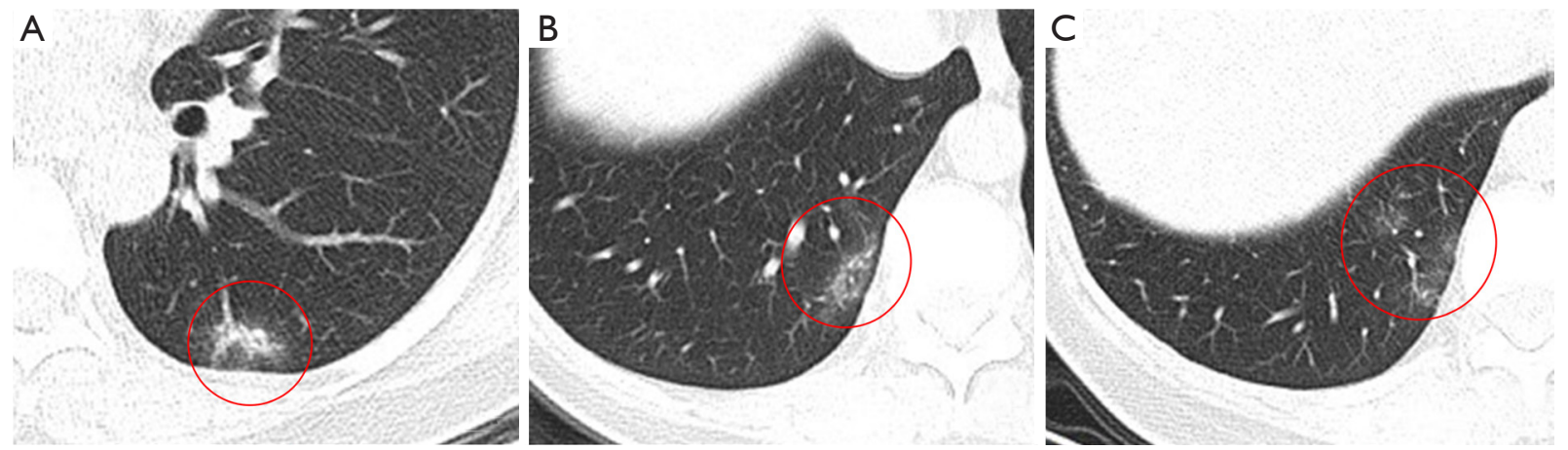

Figure 4 Patient 3, female, 43 years old. CT images showed multiple patchy GGOs under the pleura in the lower lobe of bilateral lungs, with thickened blood vessels passing through (A,B,C, red circle). GGO, ground-glass opacity.

the general incubation period is $0-14$ days with an average of about 3-5 days $(9,10)$. It was therefore most likely that the second time point of January 20 was the epidemiologic history of COVID-19 pneumonia outbreaks in this family. We speculate that Patient 3 may be the source of infection in the family, and she may have asymptomatically transmitted 2019-nCoV to the 4 other family members.

Patients with COVID-19 pneumonia can be asymptomatic. Shi et al. analyzed the clinical and CT data of 81 patients with COVID-19 pneumonia, and found 

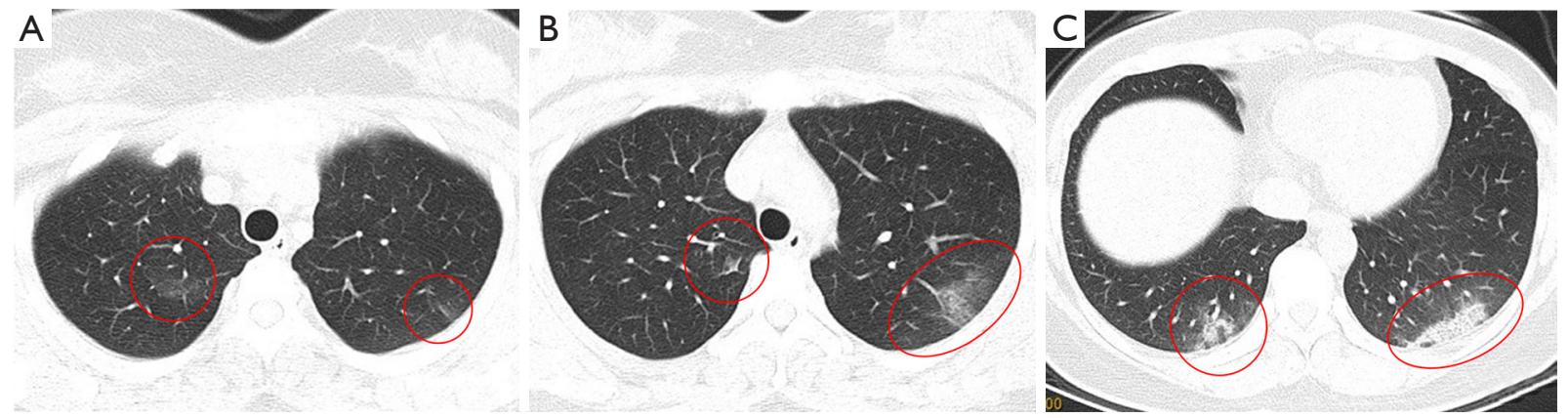

Figure 5 Patient 4, female, 41 years old. CT images showed multiple patchy GGOs in the upper lobe and lower lobe of bilateral lungs, with thickened blood vessels passing through (A,B,C, red circles). GGO, ground-glass opacity.

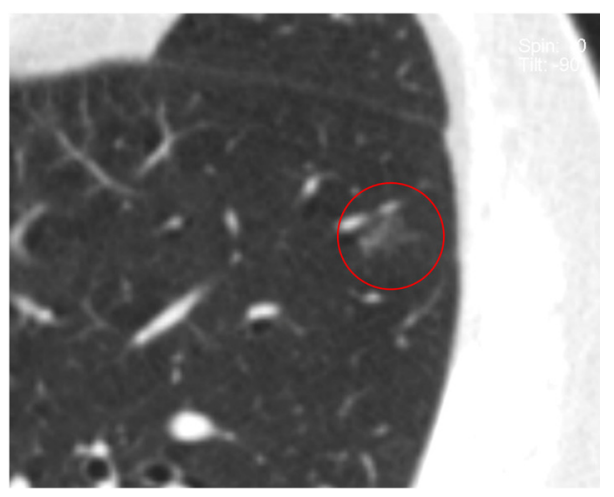

Figure 6 Patient 5, male, 45 years old. CT images showed a subsegmental GGO in the anterior basal segment of the left lower lobe (red circle). GGO, ground-glass opacity.

that 15 patients were asymptomatic (18.5\%), but lung CT results showed multiple GGO lesions (12). Four of the 5 patients diagnosed with COVID-19 pneumonia in this study did not have clinical symptoms (fever, cough, fatigue, sore throat, etc.), but GGO lesions of varying degrees were present on their lung CT scans. This finding suggested that GGO lesions in the lungs of some COVID-19 pneumonia patients appeared earlier than clinical symptoms in the early stage of onset. Several recent studies have shown human-tohuman transmission of 2019-nCoV in hospitals and home environments, and a familial cluster outbreak of COVID-19 pneumonia has draw researcher's attention $(8-10,13)$. Chan et al. reported a case of an outbreak of COVID-19 pneumonia in a Shenzhen family (8). Five of the six family members developed fever, cough, and/or diarrhea after returning to Shenzhen from Wuhan, and 2019-nCoV was positive by RT-PCR. Another 10-year-old family member who had not recently been to Wuhan was diagnosed with
COVID-19 pneumonia after several days of contact with 4 of the family members, and the "human-to-human" transmission was confirmed. In the study of Chan et al., there was one asymptomatic patient $(1 / 6,16.7 \%)$ with CT abnormalities. In both their study and our data, all infected patients in these 2 family clusters had multiple GGO lesions on their lung CT imaging.

COVID-19 patients can develop fever, cough, sore throat, fatigue, and other clinical symptoms. The most common symptoms are fever and cough $(7,9)$. Guan et al. (9) analyzed 1,099 cases of COVID-19 confirmed by RT-PCR from 552 hospitals across China and found that only $43.1 \%$ of patients had fever symptoms at the time of consultation, $67.7 \%$ had cough symptoms, and $76.4 \%$ had lung abnormalities revealed in CT imaging. Additionally, $20.9 \%$ patients have isolated $2019-\mathrm{nCoV}$ infection before or without the development of viral pneumonia. They also reported that white blood cell counts in COVID-19 pneumonia patients were reduced or normal, and lymphocyte counts in most patients were decreased $(9,14)$. In our study, the absolute count of lymphocytes was within the normal range for 5 cases, and the percentage of lymphocytes decreased in 1 case, which was similar to most patients reported in previous studies $(9,14)$. In our study, Patient 1 and Patient 5 had increased in highsensitivity C-reactive protein levels, and was transferred to a designated hospital for treatment after diagnosis. Unfortunately, after two weeks follow up, one patient's condition (Patient 1) deteriorated, one patient (Patient 5) developed cough on the second day after transfer to the designated hospital, CT results from those two patients showed increased GGO lesions. Other asymptomatic patients (Patients 2-4) were still asymptomatic, and lung abnormalities did not show progress on CT scans. Guan 
et al. (9) found that high-sensitivity C-reactive protein was significantly higher in severe patients than other patients with COVID-19 $(\mathrm{P}<0.05)$. Their study and ours suggested that high-sensitivity C-reactive protein levels may be an important laboratory indicator for patients with severe disease.

Considering the rapidly spreading epidemic of COVID-19 pneumonia, the priority was to identify any suspicious case in order to isolate the patients and administer appropriate treatment, especially when there are no scientifically proven therapies for the treatment of COVID-19 pneumonia (15). With limitations of sample collection and transportation, and kit performance, the total positive rate of RT-PCR for throat swab samples was reported to be about $30 \%$ to $60 \%$ at initial presentation (16). As a conventional imaging tool for the diagnosis of pneumonia, chest CT is relatively easy to perform and is a non-invasive and accurate rapid diagnostic tool for COVID-19 pneumonia $(17,18)$. Ai et al. $(15)$ analyzed RTPCR tests and CT scan results in 1014 patients, found that the positive rates of RT-PCR assay and chest CT imaging in their cohort were 59\% $(601 / 1,014)$, and $88 \%$ $(888 / 1,014)$ for the diagnosis of suspected patients with COVID-19, respectively. Lung CT result is one of the most important evidence for the clinical diagnosis of COVID-19 pneumonia (12). CT scans play a critical role in screening asymptomatic COVID-19 patients who was in close contact with confirmed case in a familial cluster. During the early stage of infection in patients with COVID-19 pneumonia, CT scans are more sensitive in detecting lung abnormalities than chest X-ray examination. Early CT manifestations of COVID-19 pneumonia are multiple patchy GGO lesions and consolidation charges. GGO lesions are commonly distributed in segments or sub-segments, and mostly distributed along the bronchial vascular bundle and subpleural region. Thickened blood vessels and interlobular septal thickening can be found in the GGO lesions $(4,14,19)$. The CT results of our family cluster (4 of them with asymptomatic COVID-19 pneumonia) were consistent with those of 51 patients with COVID-19 pneumonia studied by Song et al. (14). COVID-19 pneumonia needs to be distinguished from pneumonia caused by bacteria or other viruses, especially H7N9 and SARS pneumonia (17,20-22). Positive lab-tested 2019-nCoV nucleic acid is a mean to acquire a final diagnosis. Combining lung CT scan and lab-tested 2019-nCoV nucleic acid results can help early detection of asymptomatic familial cluster of COVID-19 pneumonia patients, and play a key role in the prevention and control of the epidemic.

There were a few limitations to the present study. First, the chest radiograph was not performed in the cases we studied, and it was not known whether the chest radiographs of the four asymptomatic patients could detect any abnormalities. Second, Our study has a sample of clustered outbreaks in a family, and larger sample can better reflect the true situation of asymptomatic COVID-19 pneumonia patients.

\section{Conclusions}

Asymptomatic COVID-19 pneumonia patients are found in clustered family where there is close contact with confirmed COVID-19 pneumonia family member patients, and they may be an important source of infection and an unstable factor in the outbreak. In the current COVID-19 pneumonia outbreak, asymptomatic COVID-19 pneumonia patients should not be overlooked. We believe that clustered family members who are in close contact with COVID-19 pneumonia patients need to undergo CT screening regardless of clinical symptoms. Combining lung CT scan and lab-tested 2019-nCoV nucleic acid results can help early detection of asymptomatic familial cluster of COVID-19 pneumonia patients, and play a key role in the prevention and control of the epidemic.

\section{Acknowledgments}

Funding: None.

\section{Footnote}

Data Sharing Statement: Available at http://dx.doi. org/10.21037/jtd-20-955

Conflicts of Interest: All authors have completed the ICMJE uniform disclosure form (available at http://dx.doi. org/10.21037/jtd-20-955). The authors have no conflicts of interest to declare.

Ethical Statement: The authors are accountable for all aspects of the work in ensuring that questions related to the accuracy or integrity of any part of the work are appropriately investigated and resolved. The study was conducted in accordance with the Declaration of Helsinki (as revised in 2013). This study was conducted in accordance with a protocol approved by the Research Ethics Committee 
of Zengcheng District People's Hospital of Guangzhou (No. 2020-001). Informed consent was waived due to the retrospective nature of the study.

Open Access Statement: This is an Open Access article distributed in accordance with the Creative Commons Attribution-NonCommercial-NoDerivs 4.0 International License (CC BY-NC-ND 4.0), which permits the noncommercial replication and distribution of the article with the strict proviso that no changes or edits are made and the original work is properly cited (including links to both the formal publication through the relevant DOI and the license). See: https://creativecommons.org/licenses/by-nc-nd/4.0/.

\section{References}

1. Lu R, Zhao X, Li J, et al. Genomic characterisation and epidemiology of 2019 novel coronavirus: implications for virus origins and receptor binding. Lancet 2020;395:565-74.

2. Zhu N, Zhang D, Wang W, et al. A Novel Coronavirus from Patients with Pneumonia in China, 2019. N Engl J Med 2020;382:727-33.

3. Chen H, Guo J, Wang C, et al. Clinical characteristics and intrauterine vertical transmission potential of COVID-19 infection in nine pregnant women: a retrospective review of medical records. Lancet 2020;395:809-15.

4. Pan F, Ye T, Sun P, et al. Time Course of Lung Changes at Chest CT During Recovery From Coronavirus Disease 2019 (COVID-19). Radiology 2020;295:715-21.

5. National Health Commission of the People's Republic of China: The Latest Situation of New Coronavirus Pneumonia, up to 17 February 2020. Available online: http://www.nhc.gov.cn/xcs/yqtb/202002/261f72a74be14c4 db6e1b582133cf4b7.shtml

6. Chen N, Zhou M, Dong X, et al. Epidemiological and clinical characteristics of 99 cases of 2019 novel coronavirus pneumonia in Wuhan, China: a descriptive study. Lancet 2020;395:507-13.

7. Huang C, Wang Y, Li X, et al. Clinical features of patients infected with 2019 novel coronavirus in Wuhan, China. Lancet 2020;395:497-506.

8. Chan JFW, Yuan S, Kok KH, et al. A familial cluster of pneumonia associated with the 2019 novel coronavirus indicating person-to-person transmission: a study of a family cluster. Lancet 2020;395:514-23.

9. Guan WJ, Ni ZY, Hu Y, et al. Clinical characteristics of 2019 novel coronavirus infection in China. N Engl J Med 2020;382:1708-20.
10. Li Q, Guan X, Wu P, et al. Early Transmission Dynamics in Wuhan, China, of Novel Coronavirus-Infected Pneumonia. N Engl J Med 2020;382:1199-207.

11. Rothe C, Schunk M, Sothmann P, et al. Transmission of 2019-nCoV Infection from an Asymptomatic Contact in Germany. N Engl J Med 2020;382:970-1.

12. Shi $\mathrm{H}$, Han $\mathrm{X}$, Jiang $\mathrm{N}$, et al. Radiological findings from 81 patients with COVID-19 pneumonia in Wuhan, China: a descriptive study. Lancet Infect Dis 2020;20:425-34.

13. Phan LT, Nguyen TV, Luong QC, et al. Importation and Human-to-Human Transmission of a Novel Coronavirus in Vietnam. N Engl J Med 2020;382:872-4.

14. Song F, Shi N, Shan F, et al. Emerging 2019 Novel Coronavirus (2019-nCoV) Pneumonia. Radiology 2020;295:210-7.

15. Ai T, Yang Z, Hou H, et al. Correlation of Chest CT and RT-PCR Testing in Coronavirus Disease 2019 (COVID-19) in China: A Report of 1014 Cases. Radiology 2020. doi: 10.1148/radiol.2020200642.

16. Yang $Y$, Yang $M$, Shen $C$, et al. Evaluating the accuracy of different respiratory specimens in the laboratory diagnosis and monitoring the viral shedding of $2019-\mathrm{nCoV}$ infections. medRxiv 2020. doi: https://doi.org/10.1101/20 20.02.11.20021493.

17. Hui DSC, Wong KT, Antonio GE, et al. Severe acute respiratory syndrome: correlation between clinical outcome and radiologic features. Radiology 2004;233:579-85.

18. Xie X, Zhong Z, Zhao W, et al. Chest CT for Typical 2019-nCoV Pneumonia: Relationship to Negative RT-PCR Testing. Radiology 2020. doi: 10.1148/ radiol.2020200343.

19. Lei J, Li J, Li X, et al. CT Imaging of the 2019 Novel Coronavirus (2019-nCoV) Pneumonia. Radiology 2020;295:18.

20. Koo HJ, Lim S, Choe J, et al. Radiographic and CT Features of Viral Pneumonia. Radiographics 2018;38:719-39.

21. Ooi GC, Khong PL, Müller NL, et al. Severe acute respiratory syndrome: temporal lung changes at thinsection CT in 30 patients. Radiology 2004;230:836-44.

22. Wang Q, Zhang Z, Shi Y, et al. Emerging H7N9 influenza A (novel reassortant avian-origin) pneumonia: radiologic findings. Radiology 2013;268:882-9.

Cite this article as: Liu Z, Wu Q, Zou Z, Yao Y, Cai J, Liu $\mathrm{T}$, Yang $Z$, Liu Q. Investigation of a family cluster outbreak of COVID-19 indicates the necessity of CT screening for asymptomatic family members in close contact with confirmed patients. J Thorac Dis 2020;12(7):3673-3681. doi: 10.21037/jtd-20-955 